\title{
Sex differences in clinical characteristics and I-year outcomes of young ischemic stroke patients in East China
}

This article was published in the following Dove Press journal:

Therapeutics and Clinical Risk Management

\author{
Cong Geng ${ }^{1,2, *}$ \\ Yapeng $\operatorname{Lin}^{3, *}$ \\ Qin Tang ${ }^{3}$ \\ Yifang Tang ${ }^{3}$ \\ Xiaoqing Wang ${ }^{3}$ \\ Jun-shan Zhou' \\ Jie Yang ${ }^{3}$ \\ Danni Zheng 4,5 \\ Ying-dong Zhang' \\ For the Nanjing First \\ Hospital Stroke Registry \\ Investigators
}

'Department of Neurology, Nanjing First Hospital, Nanjing Medical University, Nanjing, Jiangsu 210006 , China; ${ }^{2}$ Department of Emergency, The First Affiliated Hospital of Soochow University, Suzhou, Jiangsu 215000, China; ${ }^{3}$ Department of Neurology,

The First Affiliated Hospital of Chengdu Medical College, Xindu, Chengdu 610500, China; ${ }^{4}$ Centre for Big Data Research in Health, Faculty of Medicine, University of New South Wales, Sydney 2033, NSW, Australia;

${ }^{5}$ The George Institute for Global Health, Faculty of Medicine, University of New South Wales, Sydney 2033, NSW, Australia

*These authors contributed equally to this work

Correspondence: Jie Yang

The First Affiliated Hospital of Chengdu Medical College, 278 Baoguang Avenue, Xindu District, Chengdu 610500, China Tel +86 28830 l 7l 70

$\mathrm{Fax}+862883016069$

Email yangjieI I26@I63.com
Background: Data concerning sex differences in clinical characteristics and outcomes of young ischemic stroke (IS) patients in Eastern China are scarce. Understanding sex differences in clinical characteristics and long-term outcomes of young IS patients might provide valuable evidence for designing preventative measures and therapeutic interventions.

Methods: The study included 228 acute IS patients aged up to 50 years recruited in the prospective Nanjing First Hospital Stroke Registry over a 5-year period. Univariable and multivariable logistic regression analyses were performed to determine whether there were sex differences in clinical characteristics and outcomes of young IS patients.

Results: Admission systolic blood pressure $(130.12 \pm 24.3$ vs $137.96 \pm 24.3 \mathrm{mmHg}, P=0.005)$ of women was significantly lower than that of men. Logistic regression showed that young women had poorer outcomes defined as having modified Rankin Scale score of 3-6 at 12 months after the adjustment for history of prior stroke, NIHSS score, and complication of pneumonia (adjusted OR: 3.45; 95\% CI: 1.43-8.32).

Conclusion: Our study indicates that there may be significant differences in clinical characteristics between young women and men with acute IS in East China. Young women were more likely to be dead or dependent at 12 months after stroke onset. More attention should be paid to young women's IS prevention and management in East China.

Keywords: sex differences, young ischemic stroke, clinical characteristics, outcomes

\section{Introduction}

In China, stroke has become the leading cause of death and disability. ${ }^{1}$ Ischemic stroke (IS) has been reported to be the most common type of stroke, and its incidence in young adults has been significantly increasing. ${ }^{2}$ The long-term mortality after stroke in young adults is higher compared with expected mortality in the general population according to a recent research. ${ }^{3}$

It is of vital importance to study the characteristics and outcomes of these young Chinese stroke patients with a view toward developing tailored prevention programs. There were few studies that showed sex differences in clinical characteristics and outcomes of IS patients. ${ }^{4,5}$ Rutten-Jacobs et al assessed the survival status of 606 young IS patients and found that 20 -year mortality was higher in male than in female stroke survivors. ${ }^{3}$ On the other hand, another study revealed that young women have poorer outcomes than men after IS. ${ }^{6}$ Sex differences in young IS in China have not been previously evaluated.

We explored sex differences in clinical characteristics and 1-year outcomes of young IS patients using data from a prospective and long-term study of stroke registry in East China. 


\section{Methods}

\section{Subjects}

Consecutive patients with acute stroke were prospectively recruited from August 2004 to August 2008 in the Nanjing First Hospital Stroke Registry (NFHSR), which was a hospitalbased and prospective registry study of stroke patients with 1-year follow-up. Stroke was defined as acute IS, intracerebral hemorrhage, or subarachnoid hemorrhage, and the diagnosis was made according to the WHO criteria and confirmed by brain computed tomography or magnetic resonance imaging scans. ${ }^{7}$ Patients diagnosed with stroke within 14 days of onset were eligible to be enrolled and were followed up for 1 year. In the current study, IS patients aged 18-50 years were included if baseline information and data of 12-month follow-up were available. ${ }^{3}$ This study was approved by the Ethics Committee of Nanjing First Hospital and conducted in accordance with the Declaration of Helsinki, and we obtained written informed consent from all participants or their guardians.

\section{Data collection}

All IS patients were managed according to standard stroke care guidelines in the neurology department of Nanjing First Hospital. In the present study, we collected baseline patient information including age, sex, onset-to-door time (ODT), pre-stroke dependency, stroke severity, laboratory tests (admission hemoglobin, blood glucose, triglyceride, and cholesterol), brain imaging data, as well as other risk factors for IS. Pre-stroke dependency was defined as having a modified Rankin Scale (mRS) score of 3-5. ${ }^{8}$ The severity of IS was measured by National Institutes of Health Stroke Scale (NIHSS) and Glasgow Coma Scale (GCS). ${ }^{9}{ }^{10}$ Having a GCS score of $<9$ indicated serious disturbance of consciousness. Other risk factors for IS included the history of hypertension (defined as self-reported history of hypertension, or taking oral antihypertension drugs), diabetes mellitus (defined as self-reported history of diabetes mellitus, or taking hypoglycemic agents), hyperlipidemia (defined as self-reported history of hyperlipidemia, or taking oral antihyperlipidemia drugs), atrial fibrillation (defined as history of atrial fibrillation, confirmed by at least one electrocardiogram, or the presence of the arrhythmia after admittance), coronary heart disease, and prior stroke (defined as a confirmed medical history of IS or intracerebral hemorrhage), current smokers (defined as patients who had smoked $>1$ cigarette/day for over 1 year), and current drinkers (defined as patients who had consumed alcohol $>50 \mathrm{~mL} /$ day for at least 1 year). In addition, patients' systolic and diastolic blood pressure (SBP and DBP) at admission and the in-hospitalization complications were also recorded.

\section{Outcomes}

Primary outcomes at 12 months after enrollment were death or dependency as evaluated by the mRS and stroke recurrence. Death or dependency was prospectively evaluated through telephone follow-ups. Death was defined as all-cause cumulative mortality. Dependency was defined as having a mRS score of 3-5. Stroke patients were also followed prospectively to assess the recurrent stroke rate.

\section{Statistical analyses}

A comparison of categorical variables was conducted using chi-squared test. Normally distributed continuous variables including age, blood pressure, and hemoglobin were assessed by Student's $t$-test, and variables with skewed distribution, for example, ODT, GCS score, and NIHSS score at admission were compared using Wilcoxon test. The association between sex and clinical outcomes at 12 months was ascertained by univariable and multivariable logistic regression models. All variables with $P \leq 0.2$ in the univariate analyses were included in the multivariate model. SPSS version 16.0 (SPSS Inc., Chicago, IL, USA) was used for all statistical analyses. A two-sided $P$-value of $<0.05$ was considered statistically significant.

\section{Results}

\section{Baseline characteristics, complications, and outcome of young patients}

Figure 1 shows the patient selection flowchart. Over the 5-year study period, 3,412 consecutive acute cerebrovascular

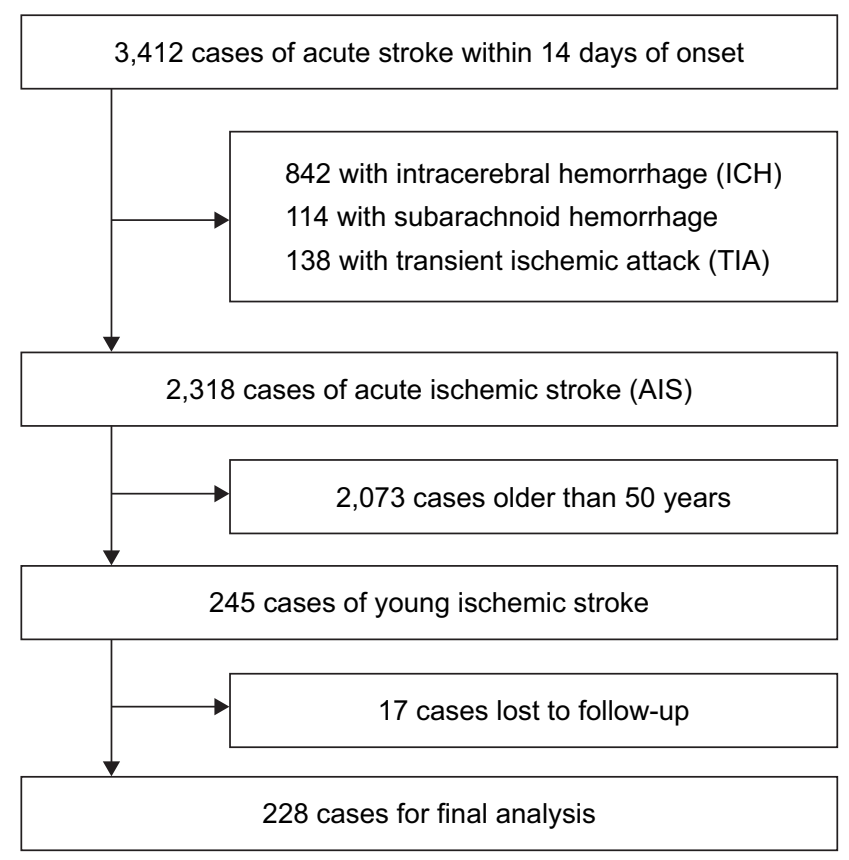

Figure I Flowchart of patient selection. 
disease patients within 14 days of onset were included in NFHSR. After exclusion of patients who were diagnosed with intracerebral hemorrhage, subarachnoid hemorrhage, and transient ischemic attack, 2,318 cases of IS were followed up for 12 months. There were altogether 245 young IS patients aged from 18 to 50 years. Seventeen $(6.9 \%)$ cases were lost to follow-up, and the remaining 228 (93.1\%) patients (81 women [35.5\%] and 147 men [64.5\%]) were included for the present analyses.

The mean age of all patients was $42.61 \pm 6.2$ years, and it was not significantly different between women and men (42.31 \pm 6.6 vs $42.78 \pm 6.0$ years, $P=0.563$ ). Table 1 demonstrates the sex-specific characteristics and outcome of young patients with IS. Compared with men, women had lower SBP (130.12 \pm 24.3 vs $137.96 \pm 24.3 \mathrm{mmHg}, P=0.005$ ),
DBP $(81.30 \pm 15.4$ vs $85.92 \pm 16.7 \mathrm{mmHg}, P=0.004)$, hemoglobin $(129.60 \pm 14.9$ vs $147.96 \pm 16.3 \mathrm{~g} / \mathrm{L}, P<0.001)$, and triglyceride $(1.61 \pm 0.8$ vs $1.97 \pm 1.6 \mathrm{mmol} / \mathrm{L}, P=0.047)$ and lower GCS score on admission $(15[14,15]$ vs $15[15,15]$, $P=0.043)$. In addition, hypertension $(19.8 \%$ vs $34.0 \%$, $P=0.013)$ was less frequent in women than in men, whereas atrial fibrillation $(14.8 \%$ vs $5.4 \%, P=0.006)$ and severe stroke (defined as having NIHSS score $>14 ; 17.3 \%$ vs $5.4 \%, P=0.003)$ were more frequent in women. Furthermore, women were significantly less likely to smoke (5.0\% vs $51.7 \%, P<0.001)$ and $\operatorname{drink}(2.5 \%$ vs $37.4 \%, P<0.001)$ than men. Regarding outcome, women had significantly higher proportion of death or dependency $(28.4 \%$ vs $11.6 \%$, $P=0.001)$ and recurrent stroke $(7.4 \%$ vs $1.4 \%, P=0.025)$ at 12 months than men.

Table I Sex differences of baseline characteristics, complications, and outcome

\begin{tabular}{|c|c|c|c|}
\hline Variables & Female $(\mathbf{N}=\mathbf{8} \mathrm{I})$ & Male $(\mathrm{N}=147)$ & $P$-value \\
\hline \multicolumn{4}{|l|}{ Baseline characteristics } \\
\hline Age (years) & $42.31 \pm 6.6$ & $42.78 \pm 6.0$ & 0.563 \\
\hline Hypertension & $16(19.8)$ & $50(34.0)$ & 0.013 \\
\hline $\mathrm{SBP}$ at admission $(\mathrm{mmHg})$ & $130.12 \pm 24.3$ & $137.96 \pm 24.3$ & 0.005 \\
\hline DBP at admission $(\mathrm{mmHg})$ & $81.30 \pm 15.4$ & $85.92 \pm 16.7$ & 0.004 \\
\hline Hemoglobin (g/L) & $129.60 \pm 14.9$ & $147.96 \pm 16.3$ & $<0.001$ \\
\hline Diabetes mellitus & $4(5.0)$ & $13(8.8)$ & 0.259 \\
\hline Random blood glucose & $6.24 \pm 2.7$ & $6.50 \pm 4.4$ & 0.596 \\
\hline Hyperlipidemia & $\mathrm{I}(\mathrm{I} .0)$ & $8(5.4)$ & 0.052 \\
\hline Triglyceride (mmol/L) & $1.61 \pm 0.8$ & $1.97 \pm 1.6$ & 0.047 \\
\hline Cholesterol (mmol/L) & $4.4 I \pm I .0$ & $4.66 \pm 1.2$ & 0.114 \\
\hline Atrial fibrillation & $12(14.8)$ & $8(5.4)$ & 0.006 \\
\hline Coronary heart disease & $\mathrm{I}(\mathrm{I} .0)$ & $\mathrm{I}(0.7)$ & 0.696 \\
\hline Prior stroke & $5(6.2)$ & $8(5.4)$ & 0.947 \\
\hline Pre-stroke dependency ${ }^{\mathrm{a}}$ & $5(6.2)$ & $7(4.8)$ & 0.625 \\
\hline Current smokers & $4(5.0)$ & $76(5 \mid .7)$ & $<0.001$ \\
\hline Current drinkers & $2(2.5)$ & $55(37.4)$ & $<0.001$ \\
\hline Median OTD time (hours) & $48[20,120]$ & $48[10.5,120]$ & 0.466 \\
\hline Median GCS score ${ }^{b}$ & $15[14,15]$ & $15[15,15]$ & 0.043 \\
\hline GCS score $<9$ & $6(7.4)$ & $5(3.4)$ & 0.104 \\
\hline Median NIHSS score ${ }^{c}$ & $4[2,10]$ & $4[1,8]$ & 0.052 \\
\hline NIHSS score $>14$ & $14(17.3)$ & $8(5.4)$ & 0.003 \\
\hline \multicolumn{4}{|l|}{ In-hospitalization complications } \\
\hline Gastrointestinal hemorrhage & $0(0)$ & $4(2.7)$ & 0.086 \\
\hline Urinary tract infection & $2(2.5)$ & $2(1.4)$ & 0.579 \\
\hline Pneumonia & $8(9.9)$ & $17(11.6)$ & 0.683 \\
\hline \multicolumn{4}{|l|}{ Outcome at 12 month } \\
\hline Death or dependency & $23(28.4)$ & $17(11.6)$ & 0.001 \\
\hline
\end{tabular}

Notes: Data are mean \pm SD, median [IQR] or $n$ (\%). aPre-stroke dependency is defined as the modified Rankin Scale scores of $3-5$; ${ }^{b} G C S$ scores can range from 3 (deep coma) to 15 (healthy); 'NIHSS scores can range from 0 (healthy) to 42 (coma with quadriplegia).

Abbreviations: DBP, diastolic blood pressure; GCS, Glasgow Coma Scale; IQR, interquartile range; NIHSS, National Institutes of Health Stroke Scale; OTD, onset to door; SBP, systolic blood pressure. 
Table 2 Factors associated with death or dependency in young ischemic stroke patients at 12 months after onset

\begin{tabular}{|c|c|c|}
\hline Variables & Unadjusted OR $(95 \% \mathrm{Cl})$ & Adjusted OR $(95 \% \mathrm{Cl})$ \\
\hline \multicolumn{3}{|c|}{ Demographic data and vascular risk factors } \\
\hline Age (years) & $1.02(0.96-1.08)$ & - \\
\hline Female sex ${ }^{a}$ & $3.03(1.51-6.10)$ & $3.45(1.43-8.32)^{*}$ \\
\hline Pre-stroke dependency & $2.19(0.27-17.62)$ & - \\
\hline Prior stroke ${ }^{a}$ & $0.44(0.14-1.33)$ & $1.65(0.39-7.01)$ \\
\hline Hypertension & $1.34(0.6 \mathrm{I}-2.92)$ & - \\
\hline Diabetes mellitus & $0.61(0.19-2.01)$ & - \\
\hline Atrial fibrillation & $0.52(0.17-1.55)$ & - \\
\hline Coronary heart disease & $0.2 \mathrm{I}(0.0 \mathrm{I}-3.4 \mathrm{I})$ & - \\
\hline Hyperlipidemia & $1.5 \mathrm{I}(0.18-12.6 \mathrm{I})$ & - \\
\hline Current smoker & $1.29(0.63-2.66)$ & - \\
\hline Current drinker & $1.25(0.56-2.80)$ & - \\
\hline \multicolumn{3}{|l|}{ Clinical features at admission } \\
\hline NIHSS score ${ }^{a}$ & $1.22(|| 4-.|.3|)$ & $1.18(1.10-1.28)^{*}$ \\
\hline GCS score ${ }^{b}$ & $0.61(0.50-0.73)$ & - \\
\hline OTD time $\leq 3$ hours & $1.78(0.60-5.25)$ & - \\
\hline \multicolumn{3}{|c|}{ In-hospitalization complications } \\
\hline Pneumonia $^{\mathrm{a}}$ & $8.52(3.29-22.08)$ & $3.75(1.01-13.90)^{*}$ \\
\hline Urinary tract infection & $2.39(0.2 \mathrm{I}-26.96)$ & - \\
\hline
\end{tabular}

Notes: ${ }^{a}$ Variables were selected for the multivariate analysis as $P$-value of the variable was not more than 0.2 in univariate analysis; ${ }^{\text {TThough }}$ the $P$-value of GCS score in univariate analysis was $<0.2$, GCS score was not involved in multivariate analysis for avoidance of collinearity with NIHSS score; *Significant $P<0.05$.

Abbreviations: GCS, Glasgow Coma Scale; NIHSS, National Institutes of Health Stroke Scale; OTD, onset-to-door.

\section{Sex-specific outcomes at 12 months after IS}

Results of univariable and multivariable analyses of associations between sex and 12-month outcome are shown in Table 2. Without adjustment for potential confounders, there were significant sex differences in death or dependency after IS (OR: 3.03; 95\% CI: 1.51-6.10). After adjustment for the potential confounders including prior stroke, NIHSS score, and complication of pneumonia, we found that female sex was still an independent predictor for poor outcomes at 12 months (adjusted OR: 3.45; 95\% CI: 1.43-8.32). Other independent predictors for poor outcomes at 12 months were: NIHSS score (adjusted OR: 1.18; 95\% CI: 1.10-1.28) and pneumonia (adjusted OR: 3.75; 95\% CI: 1.01-13.90).

\section{Discussion}

Few studies in the past had explored sex differences in the outcomes of younger IS patients, and controversy still exists. Notably, there has been no knowledge about sex differences of long-term outcomes on the eastern Chinese population. We used data from the prospective, long-term and hospitalbased registry to elucidate sex differences of young adults with IS in baseline characteristics and long-term outcomes in East China.

\section{Sex differences in characteristics of young IS patients}

An extensive knowledge of stroke risk factors in the specific population is essential for the development of efficient primary and secondary preventive strategies. The consecutive patients enrolled in NFHSR were representative of the local IS patients since over $80 \%$ stroke patients in the Qinhuai District, Nanjing City were admitted to Nanjing First Hospital. However, our results with respect to clinical characteristics of the stroke patients were not entirely similar to the previous reports. ${ }^{6}, 11-14,21$

Recently, published studies were in agreement that dyslipidemia was more prevalent in young men compared with women. ${ }^{6,11-14}$ However, no significant sex difference in proportion of patients with hyperlipidemia was observed in this study. Our research demonstrated that hypertension was prevalent among young men, as reported in some studies. ${ }^{6,13,14,21}$ Our finding of higher rates of atrial fibrillation among female stroke survivors is in agreement with the results of a prior study by Wang et al. ${ }^{4}$ The reason for the higher rates of atrial fibrillation among young females in China is not clear. Less physical activity, higher BMI, more stress, and hormone difference in Chinese young female may be contributing factors, warranting further exploration. 
In this study, there was a significantly greater proportion of current smokers and drinkers in male IS patients, which was previously shown not only in China but also in Spain and Finland. ${ }^{13,6,14}$ A cross-sectional study in North-West Iran demonstrated that there was a significant difference in diabetes rates between men and women. ${ }^{21}$ However, there was no significant sex difference in the diabetes rates in our study of young IS only. These data showing gender variations in vascular risk factors emphasized the need to carry out evidence-based stroke prevention measures targeting sex-specific risk factors in young adults.

The discrepancies in study findings could be due to several reasons. First, the definition of young patient, especially age cutoff, varied in previous studies. Specifically, published studies and registries commonly defined young adults as those younger than 45 or 50 years. In addition, different research background, inclusion criteria of patients, and adoptive statistical methods could also result in discrepancy in study findings. Therefore, sex differences in emerging modifiable risk factors from various populations suggested that the prevention strategies should also differ by sex along with race and ethnicity in the younger IS group.

\section{Sex differences in 12-month outcomes after IS}

Past studies on young stroke patients have mostly focused on mortality as an outcome but not functional outcomes. ${ }^{13,15-17}$ To our knowledge, few existing studies analyzed the sex differences in functional outcomes in young IS patients after controlling for potential confounding factors. ${ }^{6,13}$ In our study, both death and dependency as outcomes at 12 months after onset were recorded. In accordance with the report by Martínez-Sánchez et al, the multivariable logistic regression analysis herein suggested that young women were more likely to be dead or dependent than men at the time of follow-up. ${ }^{6}$

In addition, our study showed that the higher NIHSS scores at admission, the worse functional outcomes at 12-month follow-up, similar to those in previous series. ${ }^{6,13,18,19}$ Young women had a higher prevalence of atrial fibrillation in our study, which caused more recurrent stroke and cardiogenic stroke in our study. Compared with other causes of stroke, cardiogenic stroke had worse NIHSS at presentation and worse outcomes after stroke. Moreover, young patients complicated with pneumonia would have higher prevalence of death or dependency after the adjustment for sex, prior stroke, and NIHSS score. Heikinheimo et $\mathrm{al}^{20}$ reported a study of 681 IS patients aged 15-49 years and found that poststroke infections comprising mostly pneumonia were associated with unfavorable short-term outcomes and higher long-term mortality, which is consistent with our research. Taken together, these results of data analysis reflect that aggressive management of severe female patients and prevention of pulmonary infection after stroke are of great importance.

\section{Strengths and limitations}

Our study was conducted using hospital-based cohort data with strengths including its prospective design, long-term follow-up, high follow-up rate, and good representation of local young patients with IS. The complete clinical characteristics and 12-month follow-up data enabled us to investigate sex differences of young adults with IS, which was not explored previously in East China.

However, there were some limitations in present study as described below. NFHSR is a single-center and teaching hospital-based stroke registry in Nanjing, the number of patients included is relatively small, and not all young IS patients in East China were included, which might bias the results. Also, potential confounding factors such as patients' economic status, in-hospital special management (thrombectomy or intravenous thrombolysis), and posthospital management that may have influenced the sex disparity in long-term outcomes were lacking. However, special management strategies such as thrombectomy or intravenous thrombolysis may not have influenced our result as those treatments were not available in our hospital during 2004-2008. Due to the evolution of treatment options over the recent years, there might be a difference in results from more recent data (eg, prescribing of novel oral anticoagulants, intravenous thrombolysis, and mechanical thrombectomy increased, so the number of severe ISs might decrease over time).

\section{Conclusion}

Our study demonstrated that there may be significant sex differences of young IS patients in clinical characteristics and long-term outcomes in East China. Specifically, young women were more likely to be dead or dependent than men at 12-month follow-up, even after adjustment for confounding factors. More attention should be paid to the prevention and management of IS in young women in East China.

\section{Acknowledgments}

The work was supported by grants from National Natural Science Foundation of China (81471199), China Youth Clinical Research Fund (2017-CCA-VG-029), and Sichuan Science and Technology Program (2018JY0026). The funders had no role in the planning of the study or interpretation of the results. We would like to also express our 
sincere gratitude to the patients and their families who were involved in this registry study, and the clinical and research teams of the neurology department. The abstract of this paper was presented as a poster at the 2017 Asia-Pacific Stroke Conference as a poster; Oct 26-29, 2017; Nanjing. It was published in "Poster Abstracts" in Cerebrovascular Diseases 2017;44(Suppl 1):1-52 DOI: 10.1159/000481589.

\section{Disclosure}

The authors report no conflicts of interest in this work.

\section{References}

1. Liu L, Wang D, Wong KS, Wang Y. Stroke and stroke care in China: huge burden, significant workload, and a national priority. Stroke. 2011; 42(12):3651-3654.

2. Smajlović D. Strokes in young adults: epidemiology and prevention. Vasc Health Risk Manag. 2015;11:157-164.

3. Rutten-Jacobs LC, Arntz RM, Maaijwee NA, et al. Long-term mortality after stroke among adults aged 18 to 50 years. JAMA. 2013;309(11): $1136-1144$.

4. Wang Z, Li J, Wang C, et al. Gender differences in 1-year clinical characteristics and outcomes after stroke: results from the China National Stroke Registry. PLoS One. 2013;8(2):e56459.

5. Giralt D, Domingues-Montanari S, Mendioroz M, et al. The gender gap in stroke: a meta-analysis. Acta Neurol Scand. 2012;125(2):83-90.

6. Martínez-Sánchez P, Fuentes B, Fernández-Domínguez J, et al. Young women have poorer outcomes than men after stroke. Cerebrovasc Dis. 2011;31(5):455-463.

7. Stroke-1989. Recommendations on stroke prevention, diagnosis, and therapy. Report of the WHO Task Force on Stroke and other Cerebrovascular Disorders. Stroke. 1989;20(10):1407-1431.

8. Banks JL, Marotta CA. Outcomes validity and reliability of the modified Rankin scale: implications for stroke clinical trials: a literature review and synthesis. Stroke. 2007;38(3):1091-1096.

9. Brott T, Adams HP Jr, Olinger CP, et al. Measurements of acute cerebral infarction: a clinical examination scale. Stroke. 1989;20(7):864-870.

10. Teasdale G, Jennett B. Assessment of coma and impaired consciousness. A practical scale. Lancet. 1974;2(7872):81-84.

11. Ji R, Schwamm LH, Pervez MA, Singhal AB. Ischemic stroke and transient ischemic attack in young adults: risk factors, diagnostic yield, neuroimaging, and thrombolysis. JAMA Neurol. 2013;70(1):51-57.

12. Putaala J, Yesilot N, Waje-Andreassen U, et al. Demographic and geographic vascular risk factor differences in European young adults with ischemic stroke: the 15 cities young stroke study. Stroke. 2012; 43(10):2624-2630.

13. Zhang B, Pu S, Zhang W, et al. Sex differences in risk factors, etiology, and short-term outcome of cerebral infarction in young patients. Atherosclerosis. 2011;216(2):420-425.
14. Putaala J, Metso AJ, Metso TM, et al. Analysis of 1008 consecutive patients aged 15 to 49 with first-ever ischemic stroke: the Helsinki young stroke registry. Stroke. 2009;40(4):1195-1203.

15. Aarnio K, Haapaniemi E, Melkas S, Kaste M, Tatlisumak T, Putaala J. Long-term mortality after first-ever and recurrent stroke in young adults. Stroke. 2014;45(9):2670-2676.

16. Greisenegger S, Zehetmayer S, Ferrari J, et al. Clinical predictors of death in young and middle-aged patients with ischemic stroke or transient ischemic attack: long-term results of the Vienna Stroke Registry: clinical predictors of ischemic stroke mortality in patients $<60$ years. J Neurol. 2011;258(6):1105-1113.

17. Putaala J, Curtze S, Hiltunen S, Tolppanen H, Kaste M, Tatlisumak T. Causes of death and predictors of 5-year mortality in young adults after first-ever ischemic stroke: the Helsinki Young Stroke Registry. Stroke. 2009;40(8):2698-2703.

18. Varona JF, Bermejo F, Guerra JM, Molina JA. Long-term prognosis of ischemic stroke in young adults. Study of 272 cases. J Neurol. 2004; 251(12):1507-1514.

19. Nedeltchev K, der Maur TA, Georgiadis D, et al. Ischaemic stroke in young adults: predictors of outcome and recurrence. J Neurol Neurosurg Psychiatry. 2005;76(2):191-195.

20. Heikinheimo T, Broman J, Haapaniemi E, Kaste M, Tatlisumak T, Putaala J. Preceding and poststroke infections in young adults with first-ever ischemic stroke: effect on short-term and long-term outcomes. Stroke. 2013;44(12):3331-3337.

21. Talebi M, Ghertasi M, Taheraghdam A, Andalib S, Sharifipour E. A comparison of risk factors and severity of ischemic stroke in female and male genders in North-West Iran: a cross-sectional study. Iran $J$ Neurol. 2018;13(4):215-219.

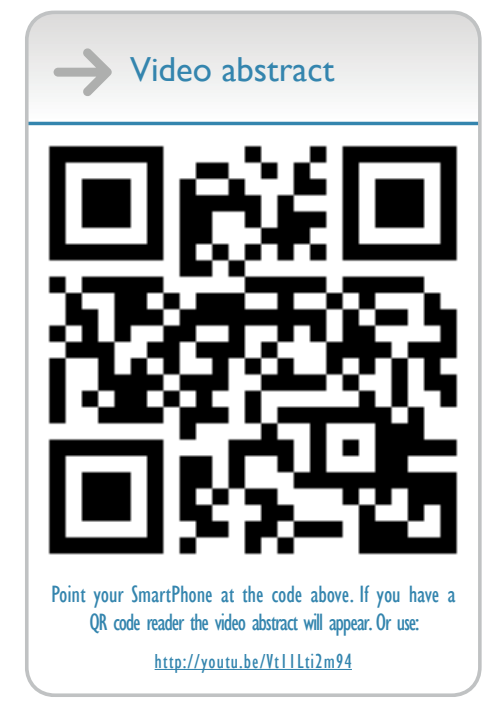

Therapeutics and Clinical Risk Management

\section{Publish your work in this journal}

Therapeutics and Clinical Risk Management is an international, peerreviewed journal of clinical therapeutics and risk management, focusing on concise rapid reporting of clinical studies in all therapeutic areas, outcomes, safety, and programs for the effective, safe, and sustained use of medicines. This journal is indexed on PubMed Central, CAS,

\section{Dovepress}

EMBase, Scopus and the Elsevier Bibliographic databases. The manuscript management system is completely online and includes a very quick and fair peer-review system, which is all easy to use. Visit $\mathrm{http}: / /$ www.dovepress.com/testimonials.php to read real quotes from published authors. 